\title{
The VWFA Is the Home of Orthographic Learning When Houses Are Used as Letters
}

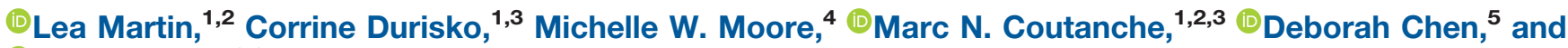 \\ (1) Julie A. Fiez ${ }^{1,2,3}$
}

https://doi.org/10.1523/ENEURO.0425-17.2019

${ }^{1}$ Department of Psychology, ${ }^{2}$ Center for the Neural Basis of Cognition, ${ }^{3}$ Learning Research and Development Center, University of Pittsburgh, Pittsburgh, PA $15260,{ }^{4}$ Department of Communication Sciences and Disorders, West Virginia University, Morgantown, WV 26506, and ${ }^{5}$ Robert Wood Johnson Medical School, Rutgers University, New Brunswick, NJ 08901

\begin{abstract}
Learning to read specializes a portion of the left mid-fusiform cortex for printed word recognition, the putative visual word form area (VWFA). This study examined whether a VWFA specialized for English is sufficiently malleable to support learning a perceptually atypical second writing system. The study utilized an artificial orthography, HouseFont, in which house images represent English phonemes. House images elicit categorybiased activation in a spatially distinct brain region, the so-called parahippocampal place area (PPA). Using house images as letters made it possible to test whether the capacity for learning a second writing system involves neural territory that supports reading in the first writing system, or neural territory tuned for the visual features of the new orthography. Twelve human adults completed two weeks of training to establish basic HouseFont reading proficiency and underwent functional neuroimaging pre and post-training. Analysis of three functionally defined regions of interest (ROIs), the VWFA, and left and right PPA, found significant pre-training versus post-training increases in response to HouseFont words only in the VWFA. Analysis of the relationship between the behavioral and neural data found that activation changes from pre-training to post-training within the VWFA predicted HouseFont reading speed. These results demonstrate that learning a new orthography utilizes neural territory previously specialized by the acquisition of a native writing system. Further, they suggest VWFA engagement is driven by orthographic functionality and not the visual characteristics of graphemes, which informs the broader debate about the nature of category-specialized areas in visual association cortex.
\end{abstract}

Key words: fMRI; language learning; left fusiform gyrus; linguistic bridge account; reading development

\section{Significance Statement}

Fluent reading recruits a portion of the brain known as the visual word form area (VWFA), but it is less well understood how malleable the VWFA remains after acquiring literacy in a native language. There is also debate about the type of visual information the VWFA can process as orthographically meaningful. We tested whether native English-speaking adults could learn a second, visually atypical writing system for English and used neuroimaging data to assess the location of any learning effects. Participants acquired basic reading ability and learning effects were found in the neural territory that underlies English reading. This suggests that the VWFA remains plastic after initial literacy and is not restricted by the visual features of a writing system.

Received December 8, 2017; accepted January 23, 2019; First published February 11, 2019.

The authors declare no competing financial interests.
Author contributions: J.A.F., M.W.M., and C.D. designed research; C.D. and D.C. performed research; M.N.C. contributed unpublished reagents/analytic tools; L.M., C.D., and J.A.F. analyzed data; L.M. wrote the paper and J.A.F., 


\section{Introduction}

Acquiring a second language in adulthood is challenging, in part because neural resources become specialized for native language processing (Tan et al., 2003; Hull and Vaid, 2007). This specialization can make it difficult to use the same neural tissue to support fluency in a second language (Mårtensson et al., 2012; Klein et al., 2014). In this article, we examined a related question: to what degree can adults acquire a second writing system for their native language? To address this question, we taught adult native English speakers a perceptually atypical artificial orthography for English. We used behavioral and functional magnetic resonance imaging (fMRI) methods to ascertain if their newly learned reading skill involved a region already specialized for reading English, the putative visual word form area (VWFA).

The VWFA is a region in the left fusiform gyrus that preferentially responds to orthographic visual stimuli (Cohen et al., 2002; McCandliss et al., 2003; Cohen and Dehaene, 2004; Szwed et al., 2011; Glezer et al., 2015; but for alternative accounts of the VWFA, see Price and Devlin, 2003; Vogel et al., 2014). This response specialization emerges with the acquisition of literacy (Saygin et al., 2016), even when native language literacy is acquired in adulthood (Dehaene et al., 2010), suggesting an absence of a "critical" period of plasticity (Bornstein, 1989).

Less is known about the degree to which the VWFA remains plastic once it has become specialized to support a native writing system, and to what extent its recruitment depends on the perceptual characteristics of a writing system. The widespread acquisition of second language literacy suggests the VWFA can support skilled reading for multiple orthographies (Tschirner, 2016). However, this apparent ease may be misleading due to the high degree of visual similarity between naturally occurring orthographies (Hirshorn and Fiez, 2014). This visual similarity may reflect the cultural evolution of writing systems to use forms that are optimized for the representational capacities of the VWFA (Dehaene, 2009), in which case, the VWFA may be poorly equipped to respond to a perceptually atypical orthography. Further, the high degree of visual similarity between natural writing systems may allow any literacy-driven specialization of the VWFA to readily transfer to another orthography, thereby overestimating the plasticity of the VWFA for orthographies that are perceptually distant from the native orthography.

M.W.M., and C.D. provided critical revisions.

This work was supported by the National Institutes of Health Grant R01 HD060388.

Acknowledgements: We thank Paul Brendel, Robert Schwartz, Brandon Carlos, and members of the Fiez Lab for their assistance and helpful discussions.

Correspondence should be addressed to Lea Martin at leamariamartin@ gmail.com.

https://doi.org/10.1523/ENEURO.0425-17.2019

Copyright (C) 2019 Martin et al.

This is an open-access article distributed under the terms of the Creative Commons Attribution 4.0 International license, which permits unrestricted use, distribution and reproduction in any medium provided that the original work is properly attributed.
A strong test of the VWFA's plasticity therefore requires acquisition of a perceptually atypical orthography by an individual whose VWFA has already been specialized by a native orthography. The need to disentangle factors that are intertwined in naturally occurring orthographies motivates the use of an artificial orthography in the present study. We build on a previously reported study that used face images as "letters" to represent English phonemes (Moore et al., 2014b). In this previous study, orthographic learning effects were observed in the left mid-fusiform cortex, but there was ambiguity whether these effects localized to the VWFA or to tissue specialized for face processing, the left fusiform face area (FFA). Thus, it remains unclear whether orthographic learning effects localize to tissue that is specialized for processing the visual characteristics of the grapheme forms (e.g., words printed with face letters to the FFA) or whether visual stimulus with orthographic functionality may induce plasticity within the VWFA, even when it has already been specialized for a perceptually typical native orthography.

To address this question, we trained English speakers to read an artificial orthography in which images of houses represent English phonemes (HouseFont). We chose houses because they are preferentially processed in a region known as the parahippocampal place area (PPA), which is spatially distant from the VWFA. The PPA's distinctiveness allows us to identify the neural tissue dedicated to processing the graphemes of our new orthography. We employed a localizer scan to functionally identify the PPA and VWFA, and pre-training and posttraining scans to isolate neural changes associated with HouseFont learning. This allowed for a clear test of whether a VWFA tuned to a native orthography (English) has the flexibility to respond to a second orthography (HouseFont), even when this second orthography uses graphemes that are highly distinctive from those used in the Roman alphabet. If the perceptual characteristics of grapheme forms drive the locus of orthographic learning, significant learning effects should be observed in the PPA. Alternatively, if the functional use of visual forms as orthographic symbols drives the locus of orthographic learning, and the neural tissue that supports this learning remains malleable, significant learning effects should be observed in the VWFA.

\section{Materials and Methods}

\section{Participants}

Fourteen University of Pittsburgh undergraduate students were originally enrolled in the study. This sample size was selected based on research showing that imaging research can achieve power of roughly $80 \%$ using a threshold of 0.05 and 12 subjects (Desmond and Glover, 2002), and results for our prior study (Moore et al., 2014b) in which significant differences in the VWFA territory were observed for between-group comparisons ( $N=11$ and $N=12$ ) of the response to a trained versus untrained orthography. One participant dropped out on the second day of training and one dropped out after having completed everything except the post-training imaging session. Data from the final sample of 12 individuals (eight 


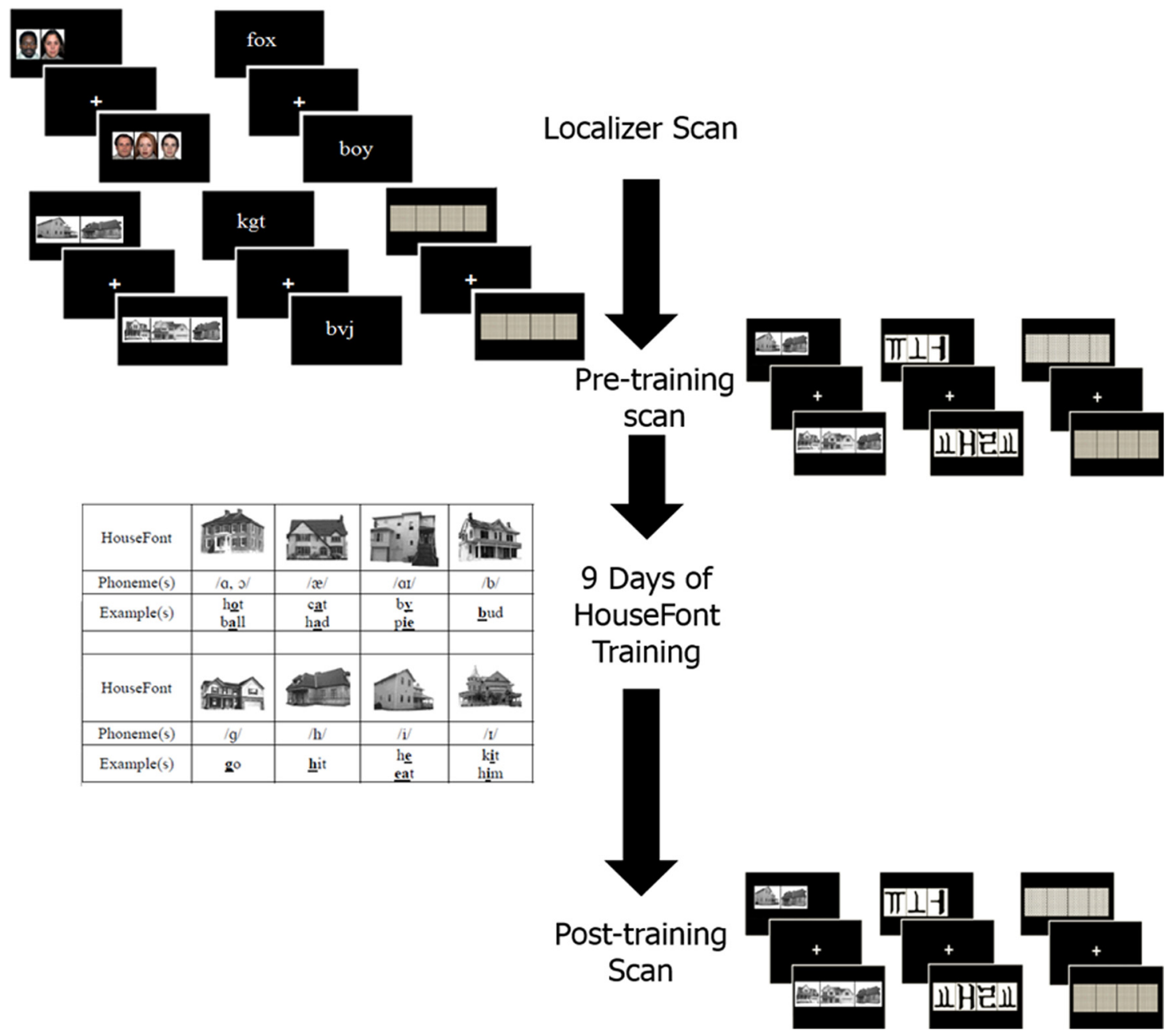

Figure 1. Participants completed a localizer scan, a pre-training scan, HouseFont training, and a post-training scan. The images alongside each point on the timeline are examples of the stimuli used for the neuroimaging sessions.

female, four male) are reported ( $\mathrm{M}$ age $=19.17$ years, SD $=1.19$ ). All participants were recruited from a database of individuals interested in participating in research studies. All study participants were right-handed, native English speakers, and had no history of second language fluency, hearing or vision issues, learning or reading problems, drug or alcohol abuse, mental illness, neurologic issues, or contraindications for fMRI. All participants provided informed consent and were compensated for their time. All procedures were approved by the institutional review board of the University of Pittsburgh.

\section{Study overview}

The study involved a two-week training protocol to learn HouseFont. Training occurred after two pre-training fMRI sessions and before a post-training fMRI session. The first of the pre-training fMRI sessions was designed to localize three regions of interest (ROIs): the VWFA and the left and right PPA. The purpose of the second pre-training fMRI session was to measure the response to words printed in HouseFont before training. The final fMRI session measured the response to HouseFont after training. Behavioral measures of post-training reading skill were also acquired as part of this final session. Participants were debriefed and paid following the post-training scan. Figure 1 provides an overview of the study timeline and the design of specific tasks. Table 1 summarizes the HouseFont training protocol. Further details are provided below.

Table 1. HouseFont training protocol

\begin{tabular}{|c|c|c|}
\hline \multirow{3}{*}{$\begin{array}{l}\text { Beek } \\
\text { Baseline }\end{array}$} & Session & Tasks \\
\hline & & Localizer fMRI \\
\hline & & Pre-training fMRI \\
\hline \multirow[t]{3}{*}{ Week 1} & Session 1 & $\begin{array}{l}\text { Phoneme training } \\
\text { Phoneme test }\end{array}$ \\
\hline & Session 2 & $\begin{array}{l}\text { Phoneme training review } \\
\text { Word level training } \\
\text { Word test (1) }\end{array}$ \\
\hline & Session 3-5 & $\begin{array}{l}\text { Word level training } \\
\text { Word test }(2-4)\end{array}$ \\
\hline \multirow[t]{2}{*}{ Week 2} & Session 6-9 & $\begin{array}{l}\text { Story level training } \\
\text { Word test }(5-8)\end{array}$ \\
\hline & Session 10 & $\begin{array}{l}\text { Reading test (GORT-4) } \\
\text { Post-training fMRI }\end{array}$ \\
\hline
\end{tabular}




\section{Pre-training $\mathrm{fMRI}$ sessions \\ Localizer session}

Participants started the study by completing a localizer fMRI session and a battery of standardized reading tests. The localizer session was conducted using a Siemens Medical Systems 3T Magnetom TIM Trio scanner with a 32-channel radio frequency coil. High-resolution structural scans were collected using an axial MPRAGE with 192 slices and 1-mm isotropic voxels. Functional data were collected across 29 interleaved slices in the same plane as the structural data ( $T R=1500 \mathrm{~ms}, \mathrm{TE}=25 \mathrm{~ms}$, $\mathrm{FOV}=200 \mathrm{~mm}, \mathrm{FA}=70^{\circ}$ ).

During functional data acquisition, participants completed a 1-back task with five categories of visual stimuli: (1) houses, (2) faces, (3) words, (4) letter-strings, and (5) patterns (Fig. 1). Following similar localizer protocols used in prior studies (Fox et al., 2009; Rossion et al., 2012), stimuli were drawn from sets of 40 exemplars for each of the non-orthographic (houses, faces, and patterns) categories, and sets of 157 exemplars for the orthographic (word and letter-string) categories. The scan consisted of four functional runs each lasting 6 min. Every run had a total of 15 blocks (three of each category, randomly ordered). Blocks consisted of 15 trials, with the stimulus for each trial presented for $200 \mathrm{~ms}$ followed by an $800-\mathrm{ms}$ fixation cross. Participants were asked to press a key when they detected a stimulus that repeated the one shown previously (i.e., 1-back). A 1-back target was presented for $12.5 \%$ of each block. A 9-s baseline condition followed each block. During this baseline, participants attended to a fixation cross at the center of the screen. During each run, the sets of house, face, and pattern stimuli were distributed pseudorandomly within each of the three blocks for each condition. With the exception of 1-back trials, the word and letter-string stimuli did not repeat. None of the house images used in the localizer task were used as stimuli in the subsequent parts of the study.

\section{Pre-training session}

The pre-training scan was completed within a week of the localizer session. For logistic reasons, the scanner, a 3T Siemens Allegra equipped with a standard radio frequency coil, differed from that used for the localizer session. High-resolution structural scans were collected using a sagittal MPRAGE with 192 slices and 1-mm isotropic voxels. Functional data were collected across 38 interleaved slices $(3.125 \times 3.125 \times 3.2 \mathrm{~mm}$ voxels $)$ parallel to the anterior-posterior commissure $(T R=2000 \mathrm{~ms}$, $\mathrm{TE}=25 \mathrm{~ms}, \mathrm{FOV}=200 \mathrm{~mm}, \mathrm{FA}=70^{\circ}$ ).

During the pre-training scan participants passively viewed 140 words printed in HouseFont and an untrained artificial orthography, KoreanFont. KoreanFont is an artificial alphabetic orthography that borrows graphemes from Hangul, the Korean writing system, and assigns them to English phonemes. They also saw 16 pattern displays that were repeated over 140 trials. Word and pattern stimuli were matched for length. Participants completed two runs, which consisted of seven blocks of each stimuli type for a total of 21 blocks. Each block contained 10 trials of the same stimulus type. For each trial, participants saw one HouseFont or KoreanFont word or pattern set for $1500 \mathrm{~ms}$, followed by $500 \mathrm{~ms}$ of a centrally located fixation cross (Fig. 1). They were instructed to attend to the stimuli, but were not asked to perform an overt task. The same set of HouseFont words were presented during the pre-training and post-training sessions; individuals were not exposed to this set of HouseFont words at any other time.

\section{HouseFont training}

HouseFont consists of 35 grapheme-to-phoneme mappings, where each grapheme is a particular house image that is used to represent a single phoneme or (in a few cases) two very similar sounds (e.g., /a/ in hot and $/ \mathrm{\rho} /$ in ball). All of the house images used for HouseFont were $300 \times 300$ pixels, normalized, and lightened to a light gray. Participants were trained to read HouseFont across nine sessions, which were broken into three phases: house-phoneme mapping (session 1), word-level training (sessions 2-5), and story-level training (session 6-9). Each training session lasted from 1 to $2 \mathrm{~h}$. These training phases are summarized.

\section{Session 1: house-phoneme mapping}

Participants began their training by learning to map each HouseFont grapheme with a corresponding phoneme using a self-paced computer program. The 35 house graphemes were visually presented in random order, and participants pressed a spacebar to hear the corresponding sound after each grapheme was displayed. Participants completed five cycles of the phoneme training, followed by a test of their ability to produce the phoneme associated with each grapheme. Participants who achieved $<90 \%$ accuracy repeated the training. All participants passed in three or fewer attempts.

\section{Sessions 2-5: word-level training}

After a brief refresher on the house-phoneme mapping, participants learned how to read aloud short words printed in HouseFont. Each session of the word-level training involved reading 400 one- to two-syllable words, which were two to five phonemes in length. The same set of 400 words was used in sessions 2-5, with the word order randomized across sessions. For each trial, participants were encouraged to attempt to read the word when it appeared; they had the option to hear any individual phoneme or the entire word if necessary. At the end of each session, a computer-based, single-word-reading test was administered. Each word test consisted of three conditions presented in a block design, with the order of blocks randomized across test sessions: old HouseFont words (words included in word-level training), new HouseFont words, and pronounceable HouseFont non-words. There were 20 trials per condition. A trial consisted of a one-syllable word that was three to four phonemes in length. The pronunciation accuracy was scored for each item, and reading latency was measured from the time a word first appeared on the screen to when the participant pressed the space bar to advance to the next word. 

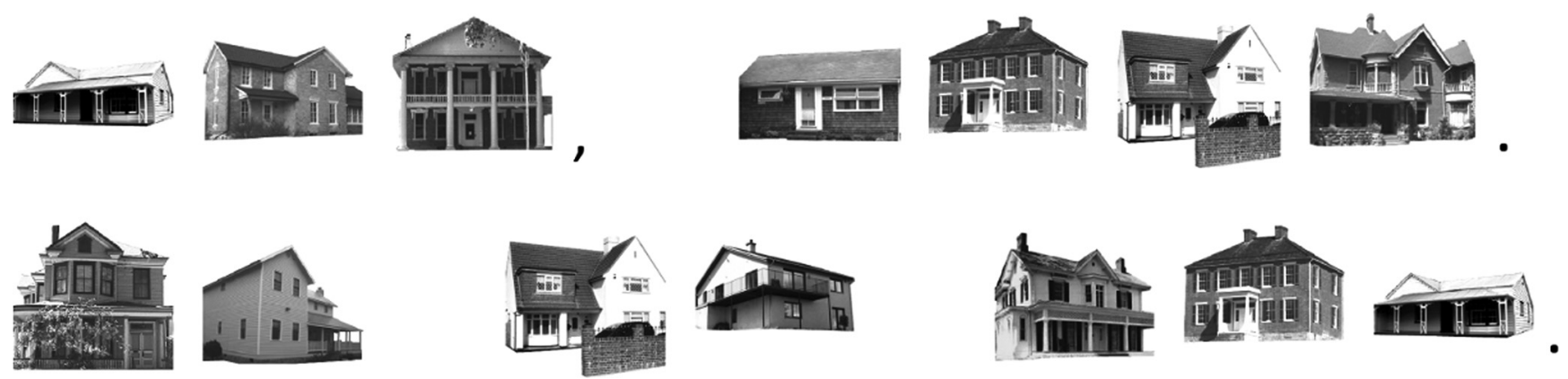

Figure 2. An example of part of a story printed in HouseFont. It reads, "Look, Father. See the ball."

\section{Sessions 6-9: story-level training}

In the final training stage, participants advanced to reading aloud short stories printed in HouseFont (Fig. 2). For each session, participants read 10 early reader stories of similar difficulty from the "Now I'm Reading!" series (Gaydos, 2003). The story level increased in difficulty with each successive session. Performance on story reading was measured by words read per minute. At the end of each session, participants completed a single-wordreading test identical in design and scoring to those used during word-level training.

\section{Post-training behavioral and fMRI session}

During the final session (session 10), participants completed behavioral testing to assess their final HouseFont reading skill and an fMRI session to measure learningrelated changes in the neural response to HouseFont. For the behavioral testing, participants' reading speed and accuracy were assessed using six passages (Form $A$ Stories 1-6) from the gray oral reading test-4 (GORT-4; Wiederholt and Bryant, 2001) that were transcribed into HouseFont. Number of words read per minute and number of errors made per word were calculated as an index of reading speed and accuracy respectively. The number of errors made per word was determined by dividing the number of errors (e.g., omissions, phoneme substitutions, whole word or part word repetitions, etc.) made by the number of words in each passage. The post-training scan was completed during session 10 immediately after administration of the behavioral tests, using the same scanner and fMRI protocol as in the pre-training scanning session.

\section{fMRI data analysis}

fMRI data preprocessing

Preprocessing of the $\mathrm{fMRI}$ data were completed using the Analysis of Functional Neurolmages (AFNI) software package (Cox, 1996). The first two brain volumes from the localizer runs and the first brain volume from the pretraining and post-training runs were removed to allow for stabilization of the signal. The functional images were slice time corrected (3dTshift), and all data were motion corrected (3dvolreg). The data were smoothed using a Gaussian filter set to a smoothing kernel of $5.5-\mathrm{mm}$ full width at half maximum. Next, the functional images were registered to the skull stripped high-resolution structural images. Images were then transformed into standard Ta- lairach space using a non-linear warping procedure in AFNI to allow for group analysis (Talairach and Tournoux, 1988). Functional images were scaled to a mean global intensity.

\section{ROIs identification}

The central question of this study is whether HouseFont learning is supported by neural tissue specialized by the acquisition of a native (English) orthography (i.e., territory at or near the VWFA) or tissue that shows selectivity for the perceptual characteristics of the non-native HouseFont orthography (i.e., the territory at or near the PPA). To address this question, the data from the localizer session were used to functionally localize a priori ROls in the left fusiform and bilateral parahippocampal cortices.

Multivariate pattern analysis (MVPA) was used to identify each of the three ROls within MATLAB using the Princeton Multi-Voxel Pattern Analysis toolbox (Detre et al., 2006). For this analysis, the functional data preprocessing was the same as described above, with one exception: as is common in MVPA, the data were not spatially smoothed (Mur et al., 2009). MVPA has been found to be more sensitive to fine grain differences between stimuli (for review, see Coutanche, 2013). This increased sensitivity allowed us to successfully localize the left fusiform ROI using the hallmark contrast used in early work characterizing the VWFA: words and letterstrings (Petersen et al., 1990; Cohen et al., 2002; Dehaene et al., 2002). To localize the PPA ROls, a house and word contrast was used.

For each run, we $z$ scored the pre-processed activity values for each voxel, accounting for the hemodynamic delay by shifting the condition time course by two TRs. A Gaussian naive Bayes (GNB) classifier was trained and tested on the activity patterns for the contrasts of interest (words versus letter-strings and houses versus words) using a leave-one-run-out cross-validation procedure, where each iteration was trained on data from all-but-one run (e.g., three runs), and tested on data from the held-out run. Classification performance from the iterations was averaged to give a single accuracy value. The resulting accuracy for the contrasts (where chance is $50 \%$ ) was then allocated to the central voxel of a three-voxel radius searchlight sphere, which was moved serially across the brain.

We identified the voxel with peak decoding accuracy for the words versus letter-strings contrast within AFNl's an- 
Table 2. Functionally defined ROls that were applied to the pre-training and post-training data

\begin{tabular}{|c|c|c|}
\hline Localizer ROI & $\begin{array}{c}\text { Cluster } \\
\text { size (voxels) }\end{array}$ & $\begin{array}{c}\text { Center of mass } \\
\text { coordinates }(x, y, z)\end{array}$ \\
\hline $\begin{array}{l}\text { Left parahippocampal } \\
\text { gyrus (L PPA) }\end{array}$ & 33 & $-28,-43,-7$ \\
\hline $\begin{array}{l}\text { Right parahippocampal } \\
\text { gyrus (R PPA) }\end{array}$ & 33 & $26,-43,-4$ \\
\hline Left fusiform gyrus (VWFA) & 33 & $-34,-55,-13$ \\
\hline
\end{tabular}

Coordinates are in Talairach space.

atomic mask of the left fusiform cortex and for the houses versus words contrast within anatomic masks of the left and right parahippocampal cortex for each subject. To generate the group level ROIs for the VWFA and PPAs, we created a 6-mm radius sphere centered on the location of average peak accuracy across all subjects for the respective contrast in each anatomic mask (Table 2).

\section{Analysis of behavioral and neural learning effects \\ Analysis of behavioral learning effects}

To test whether participants showed improvements in HouseFont reading during training, reading accuracy and reading speed were assessed for each of the word tests. A one-way repeated measures ANOVA was performed on the average reading latency scores for correct responses across the eight-word tests to determine whether reading speed changed over the course of training.

\section{Analysis of neural training effects}

To test whether participants showed neural changes associated with training (i.e., changes in the neural responses to HouseFont words), the pre-training and posttraining data were modeled using AFNl's 3dDeconvolve to estimate the BOLD response (average beta-weight value) for HouseFont and KoreanFont. The motion estimates from preprocessing were included as regressors of no interest. Then, we compared the resulting t-values for HouseFont and KoreanFont across the pre-training and post-training sessions, using both an ROl-based and a whole-brain (voxel-wise) group analysis.

For the ROI analysis, the VWFA and PPA ROIs identified from the localizer (Table 2) were applied to the pre- and post-training session data. Using AFNI 3dROlstats, the averaged beta weight value for the voxels within each ROI was obtained for each participant's response to HouseFont and KoreanFont before and after HouseFont training. These values were exported to IBM Statistical Package for the Social Sciences (SPSS) version 25. To determine whether there were training and $\mathrm{ROI}$ based differences in HouseFont activation, a $2 \times 2 \times 3$ repeated measures ANOVA was performed with orthography (HouseFont, KoreanFont), session (pre-training, post-training), and region (VWFA, left PPA, and right PPA) specified as withinsubject variables. It was expected that there would be a significant three-way interaction, which would suggest there was a differential change in HouseFont activation between ROls that resulted from HouseFont reading training. A significance threshold of $p<0.05$ was used, with correction for all violations of normalcy in the data.
As a complementary analysis approach, a whole-brain voxel-wise analysis was used to identify pre-training versus post-training changes in the response to HouseFont without a priori constraints. The computed $t$ values for the HouseFont versus KoreanFont contrast for each participant were contrasted across the pre-training versus posttraining sessions for each voxel using AFNI 3dClustSim, with a significance threshold of $p=0.005$ (corrected $p=$ 0.05 ) and a cluster size threshold of 60 contiguous voxels.

\section{Relationship between behavioral and neural measures}

To examine the relationship between behavioral and neural measures of learning, each participant's reading speed score from the final word test was standardized and combined with the standardized reading speed score from the GORT-4. This composite reading speed score was examined using a regression analysis, to determine whether the pre-training versus post-training change in the estimated BOLD responses within the VWFA ROI accounted for HouseFont reading speed variability.

Because the sample size of the current study is small, we performed a similar analysis that combined data from the participants in the current study $(N=12)$ with data from two participant groups reported by Moore et al. (2014b): one group that learned an artificial orthography with face images as letters (FaceFont; $N=12$ ) and one group that learned an artificial orthography with borrowed Korean graphs mapped to English phonemes (KoreanFont; $N=11$ ). For each participant from the Moore et al., study, the final reading speed was calculated in the same way as it was for HouseFont, by averaging the $z$ score of the GORT reading speed and the inverse $z$ score of the final word test reading speed. The imaging data from the Moore et al. study were acquired using the same design and scanner as in the current study, with the exception that only a post-training session was acquired, and instead of viewing HouseFont and KoreanFont words, participants viewed FaceFont and KoreanFont words. Because the data from the Moore et al., study were previously analyzed using a different software package, they were reprocessed using the same methods as in the current study.

Next, we used an $\mathrm{ROI}$ analysis to extract the average estimated BOLD response within the VWFA territory for each participant across our three groups (HouseFonttrained, FaceFont-trained, KoreanFont-trained). To avoid biasing the results by using the VWFA ROI identified using data from only the HouseFont participants, we drew on the literature to define an unbiased ROI for this acrossgroup analysis. Specifically, we used a coordinate from a recent study by Lerma-Usabiaga et al. (2018), where real words and consonant strings were contrasted to localize a specific VWFA subregion in the middle occipitotemporal sulcus (mOTS) that exhibits lexical-level orthographic selectivity, and which can be distinguished from a more posterior VWFA subregion that is more generally responsive to visual word forms (pOTS). The average peak coordinate reported by Lerma-Usabiaga et al. (2018) for their mOTS subregion were rounded to the closest whole numbers, transformed into Talairach space, and used as a center of a $6-\mathrm{mm}$ sphere $(-42,-57,-4)$. Using AFNI $3 \mathrm{dRO}$ Istats, the averaged beta weight value for the voxels 


\section{Story Level Reading}

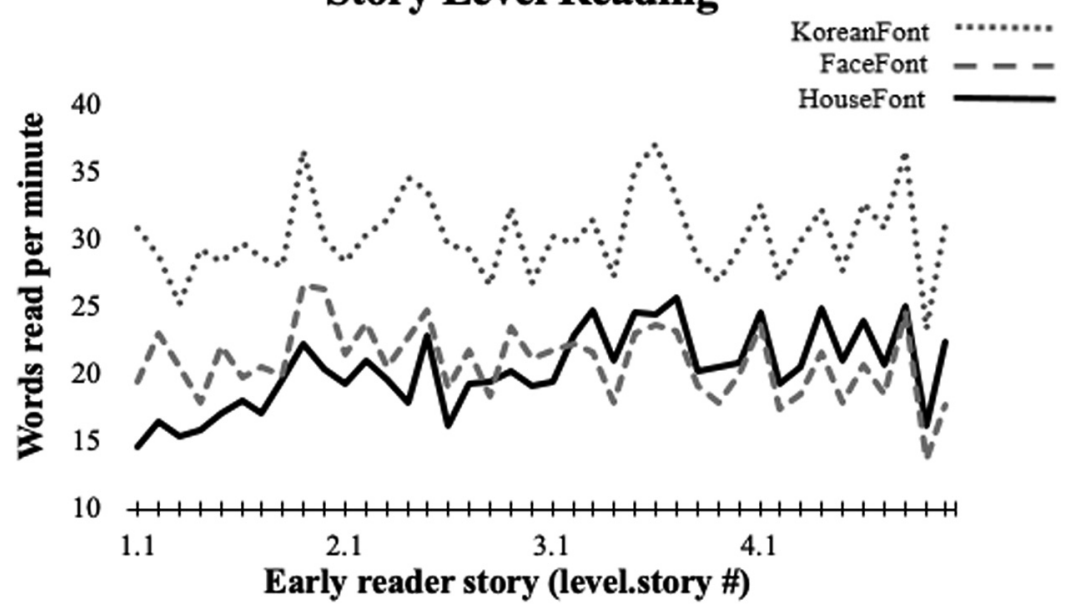

Figure 3. Stories increased in difficulty over the $4 \mathrm{~d}$ of story-level reading, but participants maintained a similar rate of words read per minute. The performance of HouseFont participants on the early reader training stories was consistent with performances seen for other artificial orthographies, KoreanFont, and FaceFont. KoreanFont and FaceFont data adapted with permission from Moore et al. (2014b).

within this mOTS ROI was obtained for each participant's response to their trained orthography during the posttraining scan. These values were entered into a regression analysis, along with the orthography learned by the participant, to predict participants' reading speed following training.

\section{Results}

\section{Behavioral measures of HouseFont learning}

Average accuracy for trained participants across all of the word tests performed during training was $90 \%$. This is not surprising, because HouseFont is a transparent orthography and so once the grapheme-phoneme mappings have been mastered, they can in theory be used to decode English words and pronounceable nonwords with perfect accuracy. For this reason, the focus of the behavioral training analyses was reading latency. To test whether participants showed improvements in HouseFont reading over the course of their training, a one-way repeated measures ANOVA was performed on the average reading latency score for correct responses on the eightword tests. Two individuals were missing a single word test and were excluded from the analysis. The Greenhouse-Geisser correction was applied because Mauchly's test of sphericity was not met, $p=0.01$. There was a significant effect of test session $F_{(2.28,20.48)}=10.47, p=$ 0.001 , which reflects a decrease in reading latencies over the course of HouseFont training. From the first word test (session 2) to the final word test (session 9), the average reading latency dropped from $6288 \mathrm{~ms}(\mathrm{SD}=1963 \mathrm{~ms})$ to $4670 \mathrm{~ms}(\mathrm{SD}=1126 \mathrm{~ms})$. This $25 \%$ reduction in reading latency indicates that participants became more skilled at reading HouseFont across the two weeks of training.

Improvements in HouseFont reading were also evident in the context of story reading. Participants maintained a relatively steady rate of reading across story level training (sessions 6-9), although the stories became increasingly more difficult across sessions (Fig. 3). By the end of story-level training (session 9), participants were reading an average of 21.85 words per minute $(S D=2.88)$. Participants also read six passages of a standardized reading assessment, the GORT, to assess final reading accuracy and speed. On this measure participants attained a mean fluency of $21.15(S D=5.13)$ words per minute, with a mean error rate of $2 \%(S D=0.02)$ per word. These proficiency results are similar to those observed for first grade children learning English (Hasbrouck and Tindal, 2006).

\section{Neural measures of HouseFont learning $\mathrm{ROI}$ analysis}

A $2 \times 2 \times 3$ repeated measures ANOVA was used to examine the effect of orthography (HouseFont, KoreanFont), session (pre-training, post-training), and region (VWFA, left PPA, and right PPA) on neural activity. This analysis revealed a main effect of orthography, $F_{(1,11)}=$ $97.07, p<0.001, \eta_{p}^{2}=0.90$, and region, $F_{(1.37,22)}=7.97$, $p=0.008, \eta_{p}^{2}=0.42$, with no effect of session, $F_{(1,11)}=$ $0.11, p=0.749, \eta_{0}^{2}=0.01$. There was a significant interaction between orthography and region, $F_{(1.79,22)}=$ $10.41, p=0.001, \eta_{p}^{2}=0.49$, and trend level interactions for orthography and session, $F_{(1,11)}=4.32, p=0.062$, $\eta_{p}^{2}=0.28$, and training and region, $F_{(1.49,22)}=3.20, p=$ $0.079, \eta_{p}^{2}=0.23$. Most importantly, the predicted threeway interaction was also significant, $F_{(1.44,22)}=6.25, p=$ $0.016, \eta_{p}^{2}=0.36$.

To examine the three-way interaction and address our a priori hypothesis that HouseFont-elicited activity in the VWFA would change after training, we ran a separate $2 \times$ 2 repeated measures ANOVA [orthography (HouseFont, KoreanFont), session [pre-training, post-training] for each region. Within the VWFA there was a main effect of orthography, $F_{(1,11)}=15.23, p=0.002, \eta_{p}^{2}=0.58$ and no effect of session, $F_{(1,11)}=0.86, p=0.374, \eta_{p}^{2}=0.07$ (Fig. 4). Critically, however, there was a significant interaction between orthography and session, $F_{(1,11)}=9.79, p=$ 

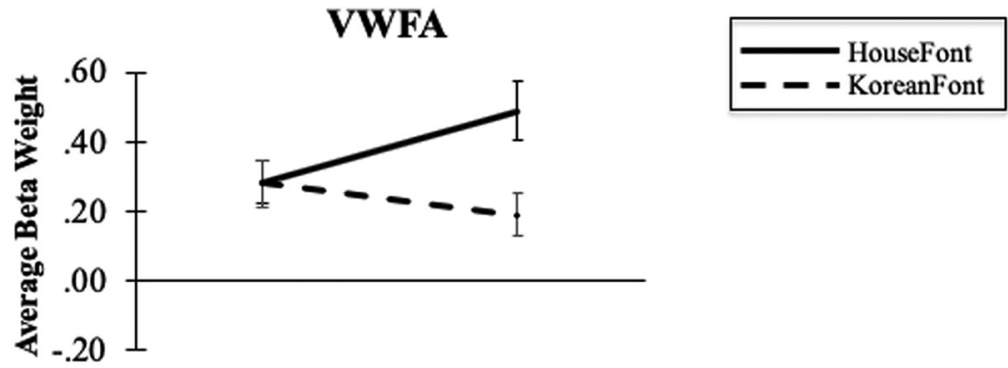

LPPA

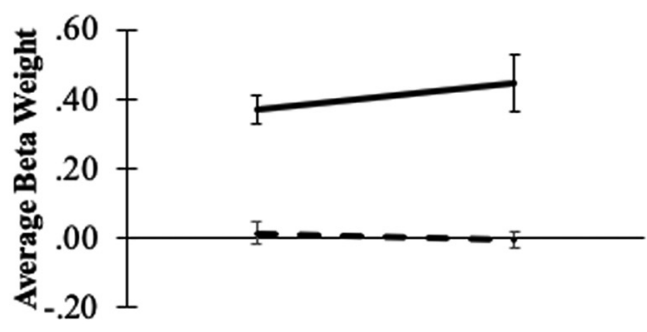

RPPA

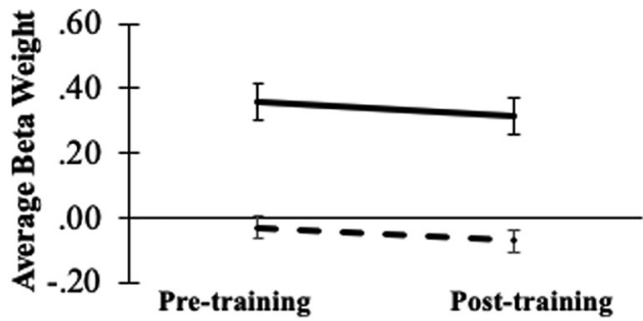

Figure 4. The VWFA showed no main effect for session or orthography, but there was a significant interaction of session and orthography. The left and right PPA showed the expected significant main effect of orthography, no main effect of training, and no significicant interaction between session and orthography. Error bars indicate SE.

$0.010, \eta_{p}^{2}=0.47$, in the VWFA. Post hoc comparisons of the interaction revealed that the response to KoreanFont decreased across sessions, $p=0.100$, while HouseFont evoked greater activation in the post-training session compared to pre-training session, $p=0.059$. These are the expected results if the HouseFont training tuned the VWFA to treat strings of HouseFont images as orthographic information.

In the left PPA, there was an effect of orthography, $F_{(1,11)}=55.43, p<0.001, \eta_{p}^{2}=0.83$, no effect of session, $F_{(1,11)}=0.47, p=0.507, \eta_{p}^{2}=0.04$, and no significant interaction between orthography and session, $F_{(1,11)}=$ 1.91, $p=0.194, \eta_{p}^{2}=0.15$. Similarly, in the right PPA, there was an effect of orthography, $F_{(1,11)}=62.12, p<$ $0.001, \eta_{p}^{2}=0.85$, no effect of session, $F_{(1,11)}=1.31, p=$ $0.276, \eta_{p}^{2}=0.11$, and no interaction between orthography and session, $F_{(1,11)}=0.00, p=0.993, \eta_{p}^{2}=0.00$. The expected main effects of orthography and the lack of other effects show that the PPA bilaterally responded more to HouseFont than KoreanFont and that HouseFont training did not alter this difference.

\section{Whole-brain voxel-wise analysis}

To investigate whether HouseFont training altered the response to HouseFont strings in areas outside of the a priori ROls, a whole-brain voxel-wise analysis was conducted with the pre-training and post-training fMRI data. HouseFont activation was compared to KoreanFont activation in both the pre-training and post-training scans separately. Then, the difference in pre-training was compared to the difference in post-training. This comparison yielded 10 significant training effect clusters, nine of which were negative, indicating more activation in posttraining. The one positive cluster, which was located in the left middle temporal gyrus (BA19), indicates more activation during pre-training (Table 3 ). Several of the clusters are in regions known to be involved in reading (Bolger et al., 2005), including the left inferior frontal gyrus, the left superior parietal lobe, and the left fusiform gyrus. Portions of the left fusiform gyrus training effect cluster overlapped with the VWFA ROI (Fig. 5), which is not surprising given the significant interaction effect found in the VWFA ROI. No training effect clusters were identified within the left or right parahippocampal gyrus.

\section{Relationship between behavioral and neural measures of HouseFont learning}

To probe the relationship between neural and behavioral measures of HouseFont learning effects, we per- 
Table 3. Clusters identified by the whole-brain voxel-wise analysis [trained orthography (HouseFont) vs untrained orthography (KoreanFont), pre-training to post-training]

\begin{tabular}{lll}
\hline \multicolumn{1}{c}{$\begin{array}{c}\text { Cluster location } \\
\text { Lize } \\
\text { Left superior parietal lobe (BA7) }\end{array}$} & $\begin{array}{c}\text { (voxels) } \\
\text { Left precentral/inferior frontal }\end{array}$ & $\begin{array}{c}\text { Peak } \\
\text { coordinates } \\
(x, y, z)\end{array}$ \\
$\quad$ gyrus (BA6/BA8) & 322 & $-28,-64,44$ \\
Right posterior cerebellum & 233 & $17,-64,-22$ \\
Left thalamus/left caudate nucleus & 197 & $-7,-13,14$ \\
Right caudate & 95 & $17,14,14$ \\
Left medial frontal gyrus (BA6) & 95 & $-1,14,44$ \\
Left middle frontal gyrus (BA46) & 81 & $-43,29,20$ \\
Left middle temporal gyrus (BA19)* & 72 & $-49,-61,17$ \\
Left fusiform gyrus (BA37) & 68 & $-40,-49,-10$ \\
Left insula (BA13) & 65 & $-31,17,11$
\end{tabular}

All clusters were identified with a corrected $p=0.05$. Coordinates are in Talairach space.

$\mathrm{BA}$, Brodmann area; * indicates the cluster that displayed more activation during pre-training.

formed a regression to test the contribution of training related activation change in the VWFA to HouseFont reading speed. A HouseFont reading speed score was calculated by averaging the $z$ score of the number of words read per minute on the GORT and the inverse $z$ score $(z$ score multiplied by -1 ) of the response time per word on the final word test. The change in activation from pretraining to post-training in the VWFA did significantly pre-

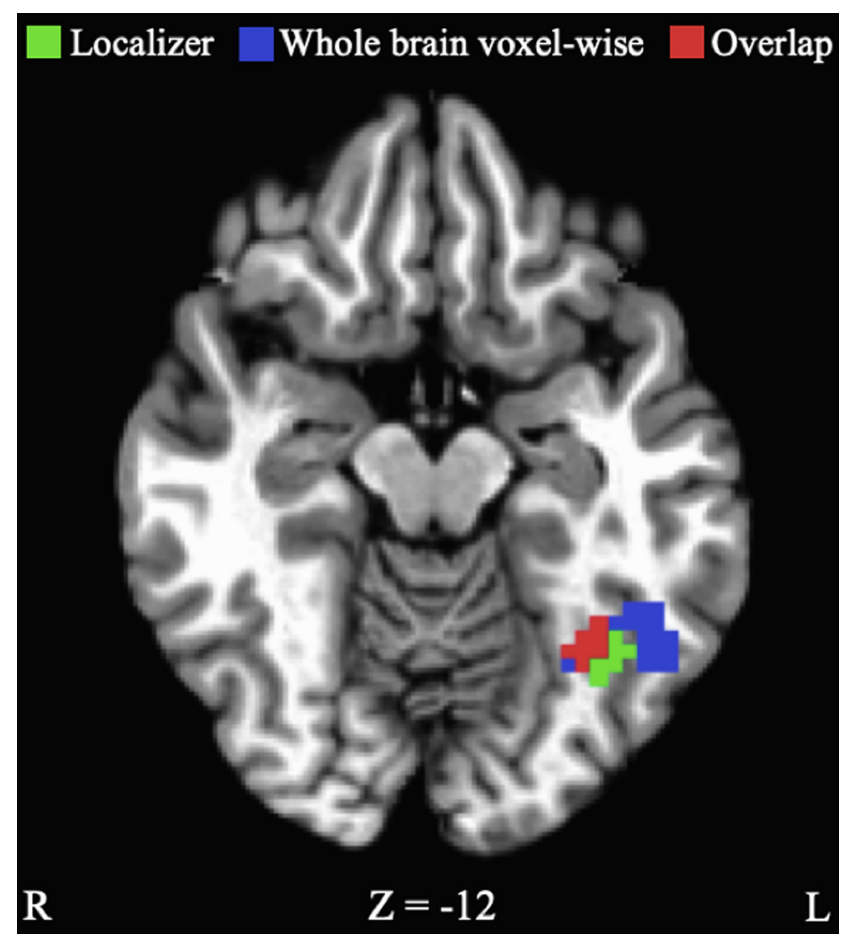

Figure 5. VWFA ROI (green) identified by the localizer scan (-34, $-55,-13$ ), and the learning effect cluster (blue) identified from the whole-brain voxel-wise analysis of activation for HouseFont versus KoreanFont from pre-training to post-training $(-40,-49$, $-10)$. Red represents the overlap. Coordinates are in Talairach space. dict reading speed $b=3.34, t_{(10)}=3.90, p=0.003$, and it explained a significant proportion of variance in reading speed scores, $R^{2}=0.60, F_{(1,10)}=15.24, p=0.003$ (Fig. 6). Based on these results, we conclude that the VWFA is critical for rapid HouseFont reading.

We obtained convergent results using data from the HouseFont-trained participants in the current study, and the FaceFont-trained and KoreanFont-trained participants previously reported by Moore et al. (2014b). While the three orthographies differ in the graphs they use and in their average reading speed (Fig. 3), we expected that behavioral measures of reading speed would be significantly predicted by the VWFA activation in response to the trained orthography. We assessed this using a specific VWFA subregion reported in the literature (mOTS; Lerma-Usabiaga et al., 2018) as an ROI (to avoid biasing our ROI localization to the HouseFont group). The posttraining response to the trained orthography within the mOTS ROI significantly predicted reading speed $b=1.38$, $t_{(32)}=2.82, p=0.008$. On the other hand, which orthography a participant learned (FaceFont, KoreanFont, or HouseFont) did not significantly predict reading speed $b$ $=-0.00, t_{(32)}=-0.01, p=0.992$. These results align with previous reports of FaceFont and KoreanFont learning effects (Moore et al., 2014b) and the findings from HouseFont. Moreover, the significant relationship between the neural and behavioral measures of learning suggest that despite the visual differences in the graphs used, reading speed variation across all three artificial orthographies can be predicted by learning effects seen within the VWFA territory (Fig. 7).

\section{Discussion}

This study tested whether acquisition of a perceptually atypical second writing system recruits the same neural tissue already tuned by native-English reading, or whether instead the locus of orthographic learning tracks with the perceptual characteristics of the grapheme forms. More specifically, we were interested in the presence or absence of artificial orthography (HouseFont) learning effects within three functionally defined areas: an orthographic area (VWFA) within the left mid-fusiform gyrus (Cohen and Dehaene, 2004), and bilateral place areas (left PPA, right PPA) within the parahippocampal gyri (Epstein and Ward, 2010). We hypothesized that orthographic learning effects would be observed in either the VWFA or the PPA, but not in both regions. Significant learning effects were found only within the VWFA, and individual differences in the magnitude of pre-training versus post-training changes in VWFA activation correlated with differences in HouseFont reading speed. We conclude the VWFA was recruited to support HouseFont literacy acquisition in our adult participants.

The results from this study converge with Moore et al. (2014b), who also observed training-related increases in the VWFA territory when participants learned one of two artificial alphabets for English: FaceFont, in which face images were used as letters, and KoreanFont, in which letters were borrowed from the Korean alphabet and mapped to English phonemes. Taken together, the results 


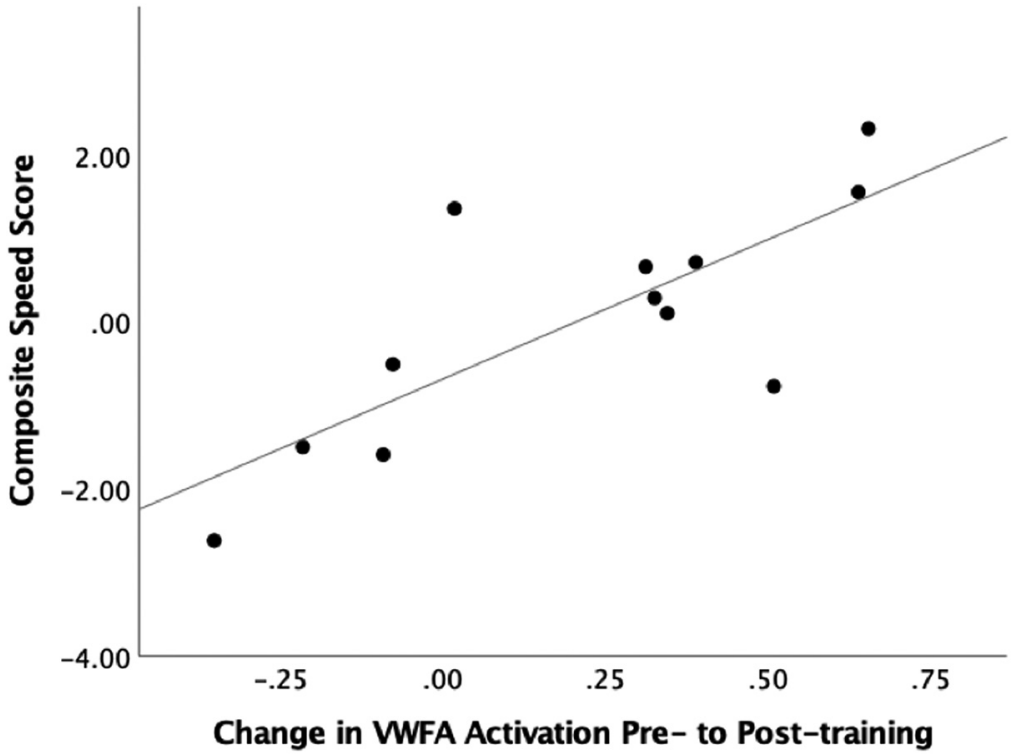

Figure 6. Scatter plot of the variance explained by the pre-training to post-training change of the VWFA for reading speed. The VWFA change showed a significant positive relationship with reading speed. Reading speed scores were zero centered.

from the current study and Moore et al. (2014b) point toward three principles of VWFA function: (1) learning a new alphabetic orthography uses VWFA tissue already specialized by acquisition of English literacy, (2) orthographies with a wide range of visual forms can induce neural plasticity in the VWFA, (3) the laterality of the VWFA is influenced by the mapping principles of an orthography.

\section{New orthographic learning uses the same tissue as English}

The HouseFont training effects demonstrate that the VWFA in native English speakers was modified by HouseFont learning. Similarly, Moore et al. (2014b) found a left-lateralized training effect for FaceFont in the vicinity of the VWFA. However, they could not conclusively assign
FaceFont learning to the same territory that supports English reading for two reasons. First, a putative left homolog of the right-lateralized face processing area (Kanwisher et al., 1997) falls in close proximity to the VWFA (Nestor et al., 2013). Consequently, the locus of observed FaceFont learning effects could arguably reflect the use of neural tissue specialized for face or orthographic processing. Second, Moore et al. (2014b) did not localize the response to printed English in their participants, so they were unable to directly compare the functional response to English and FaceFont. The present study circumvented these problems by using house graphs associated with category-specific activation in tissue that is spatially distant from the VWFA and by functionally localizing the VWFA before HouseFont training.

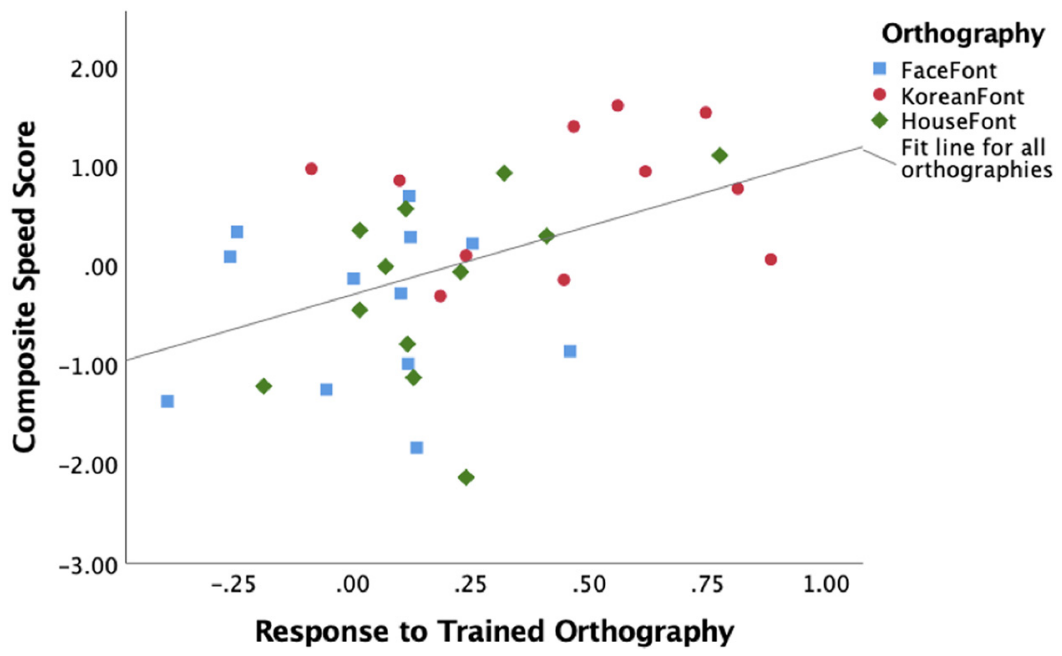

Figure 7. Scatter plot of the variance in reading speed explained by the response to trained orthography within the VWFA ROI. The response to the trained orthography showed a significant positive relationship with reading speed. Reading speed scores were zero centered across all three orthographies. 
While we attribute the change in HouseFont activation within the VWFA to orthographic learning, alternative accounts warrant consideration. It is possible that repetitive exposure to a small set of visual images could be sufficient to increase the VWFA response to the frequently experienced images. We cannot completely discount this possibility because none of our studies have involved a control group with similar exposure to the image sets in a non-literacy context. However, we favor the idea that the activation changes in the VWFA are related to literacy acquisition. This is because the regions in which activation increased were selective, the learning effects in the fusiform gyrus correlate with reading (Fig. 6; Moore et al., 2014b), and the connectivity of the VWFA is suited for visual-phonological mapping (Alvarez and Fiez, 2018).

It is also important to remember that imaging is a correlational, rather than a causal, method. It is possible that part or all of the increased VWFA activation following training could be from accessing the English orthographic representations of the HouseFont words. If this were the case, it could mean the VWFA is not necessary for accurate HouseFont reading, but rather is activated as a byproduct of accurately decoding the HouseFont word. We took extra care to ensure that HouseFont graphemes were never equated with an English grapheme and no English appeared during the training phase. Additionally, prior work with artificial orthographies found that a patient with acquired alexia was unable to learn a small set of face-phoneme pairings but was able to learn face-syllable pairings (Moore et al., 2014a). This finding suggests that the VWFA territory is critical rather collateral to learning an artificial alphabetic orthography.

\section{Visual and brain constraints on orthographic learning}

Our findings also demonstrate that there is considerable flexibility in the type of visual forms that can serve as letters of an alphabet. This is not a trivial point, as this observed flexibility is counter to some theories of how the brain and reading shape one another. Most notably, Dehaene $(2009 ; p$ 184) conjectured that orthographies have culturally evolved to be visually similar to each other because they are forced to conform to the abilities of the available neural tissue. As part of this argument, Dehaene specifically suggested that both face and house images are avoided almost entirely by writing systems because the VWFA, which supports skilled reading, is not the preferred processing area for this kind of visual information (Dehaene, 2009). The findings of this study, and those of Moore et al. (2014b), challenge this idea, because they show that participants can readily obtain basic reading proficiency for an orthography with perceptually atypical forms (house or face images).

One potentially important caveat is that individuals tend to read FaceFont and HouseFont more slowly than an artificial orthography made of more typical graphs (KoreanFont; Fig. 3). This could reflect intrinsic limitations, such as those posited by Dehaene (2009). Alternatively, it could reflect differences in the visual complexity and discriminability of faces and houses, as compared to the simpler and higher-contrast letter forms in KoreanFont, or that tissue tuned for printed English might better transfer this tuning to a visually similar orthography (e.g., KoreanFont) as compared to a visually dissimilar (e.g., FaceFont, HouseFont) orthography. Transfer effects also might occur for other characteristics of an orthography, such as its grouping of graph elements (such as the dots in Arabic words; Abadzi, 2012). This transfer effect hypothesis could be tested by comparing the learning of artificial orthographies in which graphemes are borrowed from natural orthographies varying in perceptual distance from a reader's native orthography. For example, we might predict native English speakers would read an artificial orthography with Korean graphemes more quickly than one with Arabic graphemes because Korean letters are more visually similar to English letters.

Despite baseline differences in reading speed, similar rates of learning are found across HouseFont, FaceFont, and KoreanFont (Fig. 3) and there is no evidence of a learning plateau across six weeks of training (Martin et al., 2018). Taken together, these results support Moore et al. (2014b)'s conclusion that tuning of the VWFA for English creates a "perceptual bottleneck" that slows the visual discrimination of a perceptually atypical second orthography, without preventing accurate reading and fluency gains with continued reading experience. In sum, the weight of evidence suggests that learnable orthographies are not constrained by the brain, but instead that experience with an orthography shapes the brain.

\section{Laterality effects in orthographic learning}

Finally, our results demonstrate that alphabetic orthographic learning recruits left-lateralized brain regions, regardless of the perceptual characteristics of the orthography. In the whole-brain voxel-wise analysis, a strong pattern of left-lateralized regions showed HouseFont training effects (Table 3), and a similar set of regions showed training effects in FaceFont (unpublished findings). Most notably, both the current study and Moore et al. (2014b) found training effects in the left fusiform gyrus. The lack of a training effect in the right fusiform gyrus in Moore et al. (2014b) is particularly striking as face processing has been associated with right-lateralized visual processing (Kanwisher et al., 1997; Grill-Spector et al., 2004).

HouseFont, FaceFont, and KoreanFont differ visually, but share the same alphabetic mapping principle. To clarify whether the principle of left-lateralization holds true for non-alphabetic orthographies, we turn to Hirshorn et al. (2016)'s Faceabary training study in which face images represented English syllables. The study found Faceabary training effects in both the left and right midfusiform gyrus, with more bilateral patterns of activation correlated with higher Faceabary reading fluency. In contrast, Hirshorn et al. (2016) found a strong pattern of left-lateralization outside of the fusiform gyrus when comparing pre-training to post-training activation for Faceabary, which is consistent with results from both the current study and Moore et al. (2014b). This leads us to conclude that a key driver of left-lateralized fusiform gyrus 
recruitment is whether an orthography implements an alphabetic mapping principle, while a broader leftlateralized reading network is recruited irrespective of an orthography's mapping principle.

\section{Conclusions}

The current study found that adult acquisition of a perceptually atypical alphabetic orthography induced leftlateralized neural plasticity in the VWFA. We conclude that the VWFA remains highly malleable in adulthood. Further, our results, in combination with other work, indicate that the localization of orthographic learning to the VWFA is driven by orthographic functionality rather than the visual characteristics of a script, while the lateralization of the VWFA is influenced by the mapping principles of a script.

\section{References}

Abadzi H (2012) Can adults become fluent readers in newly learned scripts? Educ Res Int 2012:8. CrossRef

Alvarez TA, Fiez JA (2018) Current perspectives on the cerebellum and reading development. Neurosci Biobehav Rev 92:55-66. CrossRef Medline

Bolger DJ, Perfetti CA, Schneider W (2005) Cross-cultural effect on the brain revisited: universal structures plus writing system variation. Hum Brain Mapp 25:92-104. CrossRef Medline

Bornstein MH (1989) Sensitive periods in development: structural characteristics and causal interpretations. Psychol Bull 105:179197. CrossRef Medline

Cohen L, Dehaene S (2004) Specialization within the ventral stream: the case for the visual word form area. Neuroimage 22:466-476. CrossRef

Cohen L, Lehéricy S, Chochon F, Lemer C, Rivaud S, Dehaene S (2002) Language-specific tuning of visual cortex? Functional properties of the visual word form area. Brain 125:1054-1069. CrossRef Medline

Coutanche MN (2013) Distinguishing multi-voxel patterns and mean activation: why, how, and what does it tell us? Cogn Affect Behav Neurosci 13:667-673. CrossRef Medline

Cox RW (1996) AFNI: software for analysis and visualization of functional magnetic resonance neuroimages. Comput Biomed Res 29:162-173. CrossRef Medline

Dehaene $S$ (2009) Reading in the brain: the new science of how we read. New York: Penguin Books.

Dehaene S, Le Clec'H G, Poline JB, Le Bihan D, Cohen L (2002) The visual word form area: a prelexical representation of visual words in the fusiform gyrus. Neuroreport 13:321-325. CrossRef Medline

Dehaene S, Pegado F, Braga LW, Ventura P, Filho GN, Jobert A, Cohen $L$ (2010) How learning to read changes the cortical networks for vision and language. Science 330:1359-1364. CrossRef Medline

Desmond JE, Glover GH (2002) Estimating sample size in functional MRI (fMRI) neuroimaging studies: statistical power analyses. J Neurosci Methods 118:115-128. CrossRef Medline

Detre GJ, Polyn SM, Moore CD, Natu VS, Singer BD, Cohen JD, Haxby JV, Norman KA (2006) The multi-voxel pattern analysis (MVPA) toolbox. Presented at the Annual Meeting of the Organization for Human Brain Mapping, Florence, Italy, November.

Epstein R, Ward E (2010) How reliable are visual context effects in the parahippocampal place area? Cereb Cortex 20:294-303. CrossRef

Fox CJ, laria G, Barton JJ (2009) Defining the face processing network: optimization of the functional localizer in fMRI. Hum Brain Mapp 30:1637-1651. CrossRef Medline

Gaydos N (2003) Playful pals. Norwalk, CT: Innovative Kids.

Glezer LS, Kim J, Rule J, Jiang X, Riesenhuber M (2015) Adding words to the brain's visual dictionary: novel word learning selec- tively sharpens orthographic representations in the VWFA. J Neurosci 35:4965-4972. CrossRef Medline

Grill-Spector K, Knouf N, Kanwisher N (2004) The fusiform face area subserves face perception, not generic within-category identification. Nat Neurosci 7:555-562. CrossRef Medline

Hasbrouck J, Tindal GA (2006) Oral reading fluency norms: a valuable assessment tool for reading teachers. Read Teach 59:636644. CrossRef

Hirshorn EA, Fiez JA (2014) Using artificial orthographies for studying cross-linguistic differences in the cognitive and neural profiles of reading. J Neurolinguistics 31:69-85. CrossRef Medline

Hirshorn EA, Wrencher A, Durisko C, Moore MW, Fiez JA (2016) Fusiform gyrus laterality in writing systems with different mapping principles: an artificial orthography training study. J Cogn Neurosci 28:882-894. CrossRef Medline

Hull R, Vaid J (2007) Bilingual language lateralization: a meta-analytic tale of two hemispheres. Neuropsychologia 45:1987-2008. CrossRef Medline

Kanwisher N, McDermott J, Chun MM (1997) The fusiform face area: a module in human extrastriate cortex specialized for face perception. J Neurosci 17:4302-4311. CrossRef Medline

Klein D, Mok K, Chen J-K, Watkins KE (2014) Age of language learning shapes brain structure: a cortical thickness study of bilingual and monolingual individuals. Brain Lang 131:20-24. CrossRef Medline

Lerma-Usabiaga G, Carreiras M, Paz-Alonso PM (2018) Converging evidence for functional and structural segregation within the left ventral occipitotemporal cortex in reading. Proc Natl Acad Sci USA 115:E9981-E9990. CrossRef Medline

Mårtensson J, Eriksson J, Bodammer NC, Lindgren M, Johansson M, Nyberg L, Lövdén M (2012) Growth of language-related brain areas after foreign language learning. Neuroimage 63:240-244. CrossRef Medline

Martin L, Hirshorn EA, Durisko C, Moore MW, Schwartz R, Zheng Y, Fiez JA (2018) Do adults acquire a second orthography using their native reading network? J Neurolinguistics. Advance online publication. Retrieved from April 16, 2018. doi: 10.1016/j.jneuroling.2018.03.004.

McCandliss BD, Cohen L, Dehaene S (2003) The visual word form area: expertise for reading in the fusiform gyrus. Trends Cogn Sci 7:293-299. CrossRef Medline

Moore MW, Brendel PC, Fiez JA (2014a) Reading faces: investigating the use of a novel face-based orthography in acquired alexia. Brain Lang 129:7-13. CrossRef Medline

Moore MW, Durisko C, Perfetti CA, Fiez JA (2014b) Learning to read an alphabet of human faces produces left-lateralized training effects in the fusiform gyrus. J Cogn Neurosci 26:896-913. CrossRef Medline

Mur M, Bandettini PA, Kriegeskorte N (2009) Revealing representational content with pattern-information $\mathrm{fMRI}$-an introductory guide. Soc Cogn Affect Neurosci 4:101-109. CrossRef Medline

Nestor A, Behrmann M, Plaut DC (2013) The neural basis of visual word form processing: a multivariate investigation. Cereb Cortex 23:1673-1684. CrossRef Medline

Petersen SE, Fox PT, Snyder AZ, Raichle ME (1990) Activation of extrastriate and frontal cortical areas by visual words and wordlike stimuli. Science 249:1041-1044. CrossRef Medline

Price CJ, Devlin JT (2003) The myth of the visual word form area. Neuroimage 19:473-481. CrossRef Medline

Rossion B, Hanseeuw B, Dricot L (2012) Defining face perception areas in the human brain: a large-scale factorial fMRI face localizer analysis. Brain Cogn 79:138-157. CrossRef Medline

Saygin ZM, Osher DE, Norton ES, Youssoufian DA, Beach SD, Feather J, Gaab N, Gabrieli JD, Kanwisher N (2016) Connectivity precedes function in the development of the visual word form area. Nat Neurosci 19:1250-1255. CrossRef Medline

Szwed M, Dehaene S, Kleinschmidt A, Eger E, Valabrègue R, Amadon A, Cohen L (2011) Specialization for written words over objects in the visual cortex. Neuroimage 56:330-344. CrossRef Medline 
Talairach J, Tournoux P (1988) Co-planar stereotaxic atlas of the human brain. 3-Dimensional proportional system: an approach to cerebral imaging. New York: Thieme.

Tan LH, Spinks JA, Feng CM, Siok WT, Perfetti CA, Xiong J, Gao JH (2003) Neural systems of second language reading are shaped by native language. Hum Brain Mapp 18:158-166. CrossRef Medline
Tschirner E (2016) Listening and reading proficiency levels of college students. Foreign Lang Ann 49:201-223. CrossRef

Vogel A, Petersen S, Schlaggar B (2014) The VWFA: it's not just for words anymore. Front Hum Neurosci 8:88. CrossRef Medline

Wiederholt JL, Bryant BR (2001) Gray oral reading tests: GORT-4. Austin, TX: Pro-Ed. 